\title{
Prenatal sound stimulation on offspring visual variation seeking'
}

\section{MICHAEL MORRA, Purdue University, Indianapolis, Ind.}

This experiment was designed to consider the effects of an aperiodic sound, delivered during the second semester of pregnancy, on offspring's responses to three levels of visual complexity. In a Greek Cross Apparatus each of 36 rat offspring was given a $6-h$ exposure to three levels of visual complexity. The results were directionally consistent with previous findings and demonstrated that the prenatally treated offspring approached all three of the stimulus patterns less frequently $(p<.001)$ and with a shorter duration $(p<.05)$ than the controls. In addition, the sound-treated animals responded differentially $(p<.005)$ to the most complex of the three visual stimuli. These findings were interpreted as having demonstrated that the treated mothers produced offspring in whom exploratory drives and specific preferences for greater visual complexity were reduced.

The purpose of this experiment was to consider the influence of an externally administered sound during pregnancy on behavioral systems other than general open-field activity. More specifically, offspring's responses to three levels of visual complexity were measured.

\section{METHOD}

The maternal hosts were 30 albino rats of the Wistar strain of approximately 90 days old at delivery. They were maintained on ad lib diet of Purina pellets and water and were housed in the colony room in which a constant 12-h dark/light cycle was controlled automatically. Sixteen litters were born of which 128 offspring survived to Day 30 . These offspring were tested in the open-field on Days 30 and 45 and the results are reported elsewhere (Morra, 1968). From the 128 offspring, 36 males were selected randomly for testing of visual variation-seeking on one day between the Days 60-75, postpartum.

The sound treatments were delivered to the maternal hosts in a box which dimensions were $24 \times 8 \times 18$ in. It was constructed from 1/2-in. plywood and elevated 2 in. from a platform. The floor and the top were constructed from 1/4-in. wire mesh and a standard door buzzer, which estimated over-all sound pressure in dB (SPL) ranged from 93-97, was attached to the outside of each of the 24-in. sides. These buzzers were placed bipolar 3 in. from the $8 \mathrm{in}$. sides and $3 \mathrm{in}$. from the base of the box.

The visual-variation seeking apparatus (VVS) was a modified Greek Cross which was similar to that reported by Denelsky \& Denenberg (1967). It was cubical in shape and constructed from sheet steel. Its internal dimensions were $(18)^{3}$ in. and its base was made from $1 / 2$-in. wire mesh. A stimulus window $(5 \times 7$ in.) was cut out of the center of each of three of the sides. They were screened with 1/2-in. wire mesh which allowed the animals to look out of the apparatus yet prevented them from leaving the apparatus. The remaining side was a hinged door used to insert and remove the animals. Uniform, diffuse, and indirect lighting was produced by a $100-\mathrm{W}$ bulb placed in a translucent light fixture $4 \mathrm{ft}$ above the center of the apparatus.

The visual variation stimuli were six cards ( $5 \times 9$ in.) which were inserted vertically $2 \mathrm{in}$. behind the screened portion of the stimulus windows. They were constructed from $1 / 8$-in. white cardboard. Three of the cards (Set 1) were black and white overlapping circles and three of the cards (Set 2) were black and white overlapping squares. Degree of visual variation was defined in terms of the number of black and white overlapping circles (or squares) per unit area. The most variable visual stimulus (MVS) cards were 1/4-in. black and white circles (or squares); the least variable visual stimulus (LVS) cards were 1 -in. black and white circles (or squares); the intermediate variable visual stimulus (IVS) cards were 1/2-in. circles (or squares).

The data for the variation-seeking trials were recorded automatically when the animals depressed a 3 in. $x 5$ in. floor panel which was located in front of each of the stimulus windows. These panels were maintained in an angularly-elevated position with a spring attachment and were pre-calibrated for each animal at $60 \mathrm{~g}$ of pressure.

At the onset of the experiment the mother rats were handled and gently stroked in a cephalocaudal direction at the rate of 10 successive strokes daily for 10 consecutive days. Each of the animals was then given a $1-\min$ trial in the open-field, weighed, marked with an ear puncher, and assigned to either the experimental or control group. These group assignments were made according to a matched group technique with the data obtained from the open-field trials.

Following these group assignments, the animals were placed in mating cages ( 3 females: 2 males) for five consecutive undisturbed days. No attempt was made to identify the onset of pregnancy in order to avoid the effects of additional handling (Ader, 1963). On Day 5 of mating the females were returned to their individual cages. On Day 7 each of the animals of the experimental group was exposed to the sound treatments at the rate of 12 successive trials daily for 5 consecutive days. Each trial consisted of a $.4 \mathrm{sec}$ sound followed by a $9.6 \mathrm{sec}$ without sound. The timing was controlled by two decade interval timers. Likewise, the animals from the control group received equal handling and time exposure to the sound apparatus without the sound.

By computing backward, the estimated days of pregnancy during the sound treatments were Days 10-17. On Day 19, the animals were given equal amounts of nesting materials and from Days 23-28, the litters were born. Subsequently, from Days 60-75, three animals tested each day for one 6 -h trial in the VVS apparatus. In this test an animal was placed in the apparatus and allowed to traverse freely to seek out the visual patterns. When an animal approached one of the stimulus windows, the spring attachment of the respective floor panel was extended thereby closing the microswitch to a channel of the event recorder. Continuous readings of frequency and duration were recorded. Both the form factor (i.e., Sets 1 or 2) and window position factor (i.e., $P_{1}, P_{2}$, or $P_{3}$ ) were systematically scheduled so that each group had equal exposure to each parameter. Food and water were readily available and were placed on the door side of the VVS apparatus.

\section{RESULTS AND DISCUSSION}

The data are found in Table 1. Parameter checks were conducted on the frequency of approach measure for both Form and Position. Analyses of variance tests on these data failed to reveal any significant preferences at the $\mathrm{p}<.05$ level.

A 2-factor analysis of variance test was conducted on the total frequency of approaches data (i.e., to all three stimulus patterns). The Experimental group approached the patterns less frequently than the Controls as follows: Sound $(F=18.41, p<.001)$; Visual Variation $(F<1.0)$; Interaction $(F<1.0)$. Likewise, orthogonal

Table 1 Visual Variation-Seeking Behaviors of Offspring

\begin{tabular}{|c|c|c|c|c|}
\hline \multirow[b]{2}{*}{ Level of Complexity } & \multicolumn{2}{|c|}{ Sound-treated } & \multicolumn{2}{|c|}{ Controls } \\
\hline & $(\mathrm{F})^{*}$ & $(\mathrm{D})^{* *}$ & $(F)^{*}$ & $(\mathrm{D})^{* *}$ \\
\hline Most variable $(1 / 4$ in.) & $\begin{array}{l}32.7 \\
(19.5)^{* * *}\end{array}$ & 10.5 & $\begin{array}{l}54.8 \\
(30.0)^{* * *}\end{array}$ & 27.7 \\
\hline Intermediate $(1 / 2$ in.) & $\begin{array}{c}35.5 \\
(18.6)^{* * *}\end{array}$ & 25.7 & $\begin{array}{l}50.7 \\
(31.3)^{* * *}\end{array}$ & 19.7 \\
\hline Least variable ( 1 in.) & $\begin{array}{c}35.1 \\
(19.4)^{* * *}\end{array}$ & 15.4 & $\begin{array}{l}59.3 \\
(22.9)^{* * *}\end{array}$ & 35.1 \\
\hline
\end{tabular}

*Frequency of approaches (means).

**Duration of time spent at each stimulus level in minutes (medians). ***Standard deviations. 
comparisons of each stimulus pattern on the duration data yielded the following U-values: Most-variable stimuli $(\mathrm{U}=246, \mathrm{z}=2.66$, $\mathrm{p}<.005)$ in which the control animals spent more time at the 1/4-in. visual stimuli; similar Mann-Whitney tests conducted to the IVS and the LVS data failed to reach the $\mathrm{p}<.05$ level.

The results suggested that sound of unknown frequency characteristics during pregnancy altered motivational systems other than open-field activity of offspring. These results seemed to indicate that both general exploratory drive as well as specific preferences for greater visual complexity were reduced.

\section{REFERENCES}

ADER, R., \& CONKLIN, P. M. Handling of pregnant rats: Effects on emotionality of their of fspring. Science, 1963, 142, 411-412.
DeNELSKY, G. Y., \& DENENBERG, V. H. Infantile stimulation and adult exploratory behavior: Effects of handling upon tactual variation seeking. Journal of Comparative \& Physiological Psychology, 1967, 63, 309-312.

FERREIRA, A. J. Emotional factors in prenatal environment. Journal of Nervous \& Mental Disease, 1965, 141, 108-118.

MORRA, M. Prenatal sound stimulation on postnatal rat open field behavior. Psychological Record, 1965, 15, 571-575.

MORRA, M. Ethanol and maternal stress on rat offspring behavior. Journal of Genetic Psychology, 1968, in press.

THOMPSON, W. R., \& WATSON, J., \& CHARLESWORTH, W. R. The effects of prenatal maternal stress on offspring behavior in rats. Psychological Monographs, 1962, 76, 1-26.

NOTE

1. These data were collected at Xavier University, Cincinnati, Ohio. 